\title{
DNA damage regulated autophagy modulator 1 recovers the function of apoptosis-stimulating of p53 protein 2 on inducing apoptotic cell death in Huh7.5 cells
}

\author{
DONGJIE LIU ${ }^{1,2^{*}}$, RUI LI ${ }^{3 *}$, XIANGHUA GUO ${ }^{1,2}$, LIJUN PANG ${ }^{1,2}$, YUNJIN ZANG ${ }^{1,4}$, \\ $\mathrm{KAI} \mathrm{LIU}^{1,2}$ and DEXI CHEN ${ }^{1,2,4}$ \\ ${ }^{1}$ Capital Medical University Affiliated to Beijing You An Hospital; ${ }^{2}$ Beijing Institute of Hepatology, \\ Beijing 100069; ${ }^{3}$ Department of Acupuncture and Mini-invasive Oncology, Beijing University of \\ Chinese Medicine Third Affiliated Hospital, Beijing 100029; ${ }^{4}$ Organ Transplantation Center, \\ The Affiliated Hospital of Qingdao University, Qingdao, Shandong 266003, P.R. China
}

Received October 13, 2016; Accepted March 2, 2018

DOI: $10.3892 / 01.2018 .8453$

\begin{abstract}
Overexpression of apoptosis-stimulating of p53 protein 2 (ASPP2) can induce apoptotic cell death in hepatoma cells, which contributes to a killing effect of ASPP2 on treating hepatocellular carcinoma (HCC). In the present study, ASPP2 overexpression failed to induce apoptotic cell death in the HCC Huh7.5 cell line, but promoted autophagy development by inhibiting AKT/mTOR pathway. Inhibition of autophagy using 3-methyladenosine recovered the function of ASPP2 on inducing apoptotic cell death, indicating that ASPP2-induced autophagy has an anti-apoptotic role in Huh7.5 cells. A previous study demonstrated that ASPP2-induced autophagy could induce apoptosis in a CHOP- and DRAM-dependent manner, in which CHOP is involved in the initiation of autophagy and DRAM allows autophagy to induce apoptosis. In the present study, CHOP and DRAM were not involved in ASPP2-induced autophagy; however, the induction of DRAM overexpression recovered the apoptosis-inducing function of ASPP2, indicating that DRAM overexpression switches the role of ASPP2-induced autophagy from anti-apoptotic to pro-apoptotic in Huh7.5 cells. Thus, in combination with DRAM, ASPP2 may better perform its pro-apoptotic role by preventing the occurrence of anti-apoptotic autophagy.
\end{abstract}

Correspondence to: Professor Dexi Chen or Dr Kai Liu, Capital Medical University Affiliated to Beijing You An Hospital, 8 XiTouTiao, Beijing 100069, P.R. China

E-mail: dexichen@ccmu.edu.cn

E-mail: liukaihj@163.com

*Contributed equally

Key words: apoptosis-stimulating of p53 protein 2, autophagy, apoptosis, damage regulated autophagy modulator 1 , hepatocellular carcinoma

\section{Introduction}

Hepatocellular carcinoma (HCC) is one of the most difficult types of cancer to treat. The current therapeutic strategies designed to induce apoptosis are not effective enough to completely eliminate HCC (1). Apoptosis-stimulating of p53 protein 2 (ASPP2) is composed of ankyrin repeats, an $\mathrm{SH} 3$ domain, and a proline-rich region (1). ASPP2 binds to p53 through its $\mathrm{C}$-terminus to stimulate the transactivation function of $\mathrm{p} 53$ on the promoters of pro-apoptotic genes (1). Other studies also demonstrated that ASPP2 could induce apoptosis in a p53-independent manner (2-4). Previous results have indicated that induction of ASPP2 overexpression can promote apoptotic cell death in hepatoma cells (such as HepG2 or Hep3B), which emphasizes the value of ASPP2 in treating HCC $(3,4)$.

Autophagy is an evolutionarily conserved pathway that aids maintenance of cellular homeostasis by targeting proteins and organelles to lysosomes for degradation (5). ASPP2 overexpression can induce autophagy by promoting the expression of the transcription factor C/EBP homologous protein (CHOP) (4). ASPP2-induced autophagy can induce apoptosis (autophagic apoptosis) in hepatoma cells in a DNA damage regulated autophagy modulator 1 (DRAM)-dependent manner (4). Induction of DRAM expression allows for the induction of apoptosis by autophagy, indicating that DRAM can switch autophagy from being anti-apoptotic to pro-apoptotic (4). However, autophagy can serve an anti-apoptotic role as the inhibition of autophagy can increase the level of apoptosis (6).

The present study assessed the function of ASPP2 overexpression on inducing autophagy and apoptosis in Huh7.5 cells, a HCC cell line, and the association between ASPP2-induced autophagy and apoptosis.

\section{Materials and methods}

Cell culture and treatment. Huh7.5 cells were grown in Dulbecco's Modified Eagle's Medium (DMEM; Invitrogen; Thermo Fisher Scientific, Inc., Waltham, MA, USA). DMEM 
was supplemented with $10 \%$ fetal bovine serum (FBS; Invitrogen; Thermo Fisher Scientific, Inc.). Cells were transfected with plasmids $(5 \mu \mathrm{g})$ encoding ASPP2, DRAM and green fluorescent protein-microtubule associated protein 1 light chain 3 (GFP-LC3) by using Lipofectamine 2000 (Invitrogen; Thermo Fisher Scientific, Inc.) for $48 \mathrm{~h}$. Small interfering RNAs (siRNAs; Santa Cruz Biotechnology, Inc., Dallas, TX, USA) were used to decrease the expression of CHOP (cat no. sc-35437) or DRAM (cat no. sc-96209). The control siRNA (cat no. sc-37007) was also purchased from Santa Cruz Biotechnology, Inc. siRNA transfections were performed at $80 \mathrm{nM}$ by reverse transfection with Lipofectamine RNAiMax (Invitrogen; Thermo Fisher Scientific, Inc.) for $48 \mathrm{~h}$, according to the manufacturer's protocol; 3-methyladenine (3-MA; $10 \mathrm{mM}$, Santa Cruz Biotechnology, Inc.) was added into the medium of Huh7.5 cells for $12 \mathrm{~h}$ at $37^{\circ} \mathrm{C}$ to inhibit autophagy. Cells were grown on glass cover slips for the TUNEL assay.

Western blot analysis. Cell lysates were subjected to western blot analysis, as described previously (3). Briefly, total cell lysates were separated by $10 \%$ SDS-PAGE, and the separated proteins were transferred to polyvinylidene difluoride membranes. The protein blots were blocked with 5\% non-fat milk for $1 \mathrm{~h}$ at room temperature and sequentially probed with specific primary antibodies and horseradish peroxidase-conjugated secondary antibodies. Primary antibodies and secondary antibodies were used at a dilution of 1:1,000 and 1:5,000, respectively. The detection of specific proteins on the blots was achieved using a Pierce ${ }^{\mathrm{TM}}$ ECL Western Blotting Substrate (Thermo Fisher Scientific, Inc.), and the results were captured on ImageQuant LAS 4000 (GE Healthcare, Chicago, IL, USA) using ImageQuant ${ }^{\mathrm{TM}}$ TL 7.0 software (GE Healthcare). The antibodies for the detection of LC3-I/II, p62, RAC serine/threonine-protein kinase (AKT), phosphorylated (p)-AKT, mechanistic target of rapamycin (mTOR), p-mTOR were purchased from Cell Signaling Technology, Inc. (Danvers, MA, USA); the antibodies for detection of $\beta$-actin, CHOP, DRAM were purchased form Abcam (Cambridge, CA, USA). Anti-ASPP2 antibody was purchased from Sigma-Aldrich (Merck KGaA). Goat anti-Mouse IgG and goat anti-Rabbit IgG secondary antibodies were purchased from Thermo Fisher Scientific Inc.

Cell death and apoptosis analysis. Cell death was quantified with the calcein acetoxymethyl ester (calcein-AM)/propidium iodide (PI) assay (Thermo Fisher Scientific, Inc.) as previously described (7). Briefly, calcein-AM $(1 \mu \mathrm{g} / \mathrm{ml})$ and PI $(1 \mu \mathrm{g} / \mathrm{ml})$ were added into the supernatant, and the cells were incubated with calcein-AM/PI for $15 \mathrm{~min}$. Apoptosis was detected using the terminal deoxynucleotidyl transferase dUTP nick-end labeling (TUNEL) assay (Promega Corporation, Madison, WI, USA) and the slides were mounted with $50 \%$ glycerol, as described previously (7). Briefly, at room temperature, the cells were fixed with $10 \%$ paraformaldehyde in PBS for $15 \mathrm{~min}$ and then incubated in 1\% Triton X-100/PBS for $5 \mathrm{~min}$. TUNEL detection solution was dropped onto the glass cover slips, and the cells were incubated at $37^{\circ} \mathrm{C}$ in the dark for $60 \mathrm{~min}$. Nuclear staining with DAPI was conducted for $5 \mathrm{~min}$ at room temperature following rinsing with PBS. Finally, the slips were mounted with $50 \%$ glycerol following rinsing with
PBS. The images of the calcein-AM/PI and TUNEL assays were obtained with an Olympus IX71 inverted fluorescence microscope (magnification, x20; Olympus Corporation, Tokyo, Japan). A quantitative cell death/apoptosis analysis was performed by counting $>1,000$ cells in each examination.

Statistical analysis. Data in the present study are the results of at least three independent experiments and are expressed as the mean \pm standard deviation. One-way analysis of variance followed by Tukey's multiple comparison test was used to evaluate the differences between the groups. $\mathrm{P}<0.05$ was considered to indicate a statistically significant difference.

\section{Results}

Overexpression of ASPP2 induces autophagy in Huh7.5 cells. Huh7.5 cells were co-transfected with plasmids encoding GFP-LC3 and ASPP2 (ASPP2-p) for $48 \mathrm{~h}$. A vector plasmid was used as a control for ASPP2 (Ctrl-p). An immunofluorescence assay determined that ASPP2 overexpression induced the development of GFP-LC3-II puncta-positive cells, indicating that ASPP2 overexpression induces autophagy in Huh7.5 cells (Fig. 1A and B). Huh7.5 cells were transfected with ASPP2-p or Ctrl-p for $48 \mathrm{~h}$ and then western blotting determined that ASPP2 overexpression also induced the development of LC3-II and reduced the expression of p62 (Fig. 1C). These data indicated that ASPP2 overexpression induced autophagy in Huh7.5 cells.

ASPP 2 overexpression induces autophagy by inhibiting AKT/mTOR pathway, but not by inducing the expression of CHOP or DRAM, in Huh7.5 cells. ASPP2 overexpression can induce autophagy by inducing the expression of CHOP and DRAM (4). In the present study, ASPP2 overexpression did not increase the expression of CHOP and DRAM in Huh7.5 cells (Fig. 2A). Additionally, knockdown of CHOP or DRAM using siRNAs did not significantly affect the level of GFP-LC3-II puncta-positive Huh7.5 cells following transfection with ASPP2-p for $48 \mathrm{~h}$ (Fig. 2B and C). Thus, in the present study, CHOP and DRAM were not involved in ASPP2-induced autophagy. Mechanistic target of rapamycin (mTOR) has been identified to be a negative regulator of autophagy development (8). When mTOR is phosphorylated by AKT, it is activated and then inhibits autophagy development. Here, western blot analysis determined that ASPP2 overexpression significantly reduced the levels of p-AKT and p-mTOR, indicating that ASPP2 overexpression induced autophagy development by inhibiting the activation of the AKT-mTOR pathway in Huh7.5 cells (Fig. 2A).

Autophagy impairs the function of ASPP2 overexpression on inducing apoptotic cell death in Huh7.5 cells. Huh7.5 cells were transfected with ASPP2-p for $48 \mathrm{~h}$ and then TUNEL and Calcein AM/PI assays were used to detect apoptosis and cell death, respectively. Notably, the TUNEL and Calcein AM/PI assays determined that ASPP2 overexpression could not induce apoptosis and cell death in Huh7.5 cells (Fig. 3A and B). Autophagy has been identified to be an anti-apoptotic factor in cells, so whether ASPP2-induced autophagy could impair the pro-apoptotic function of ASPP2 was investigated. An autophagy inhibitor, 3-MA, was used to inhibit autophagy development in 
A

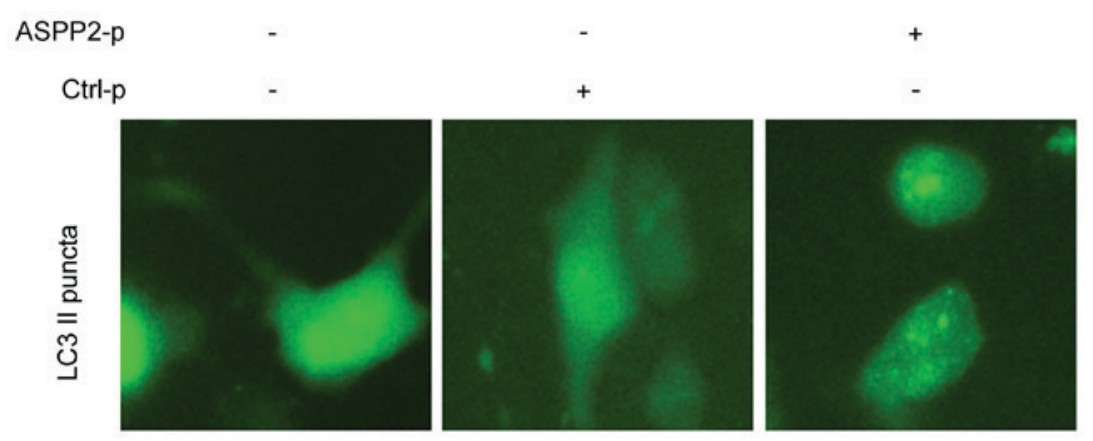

B



C

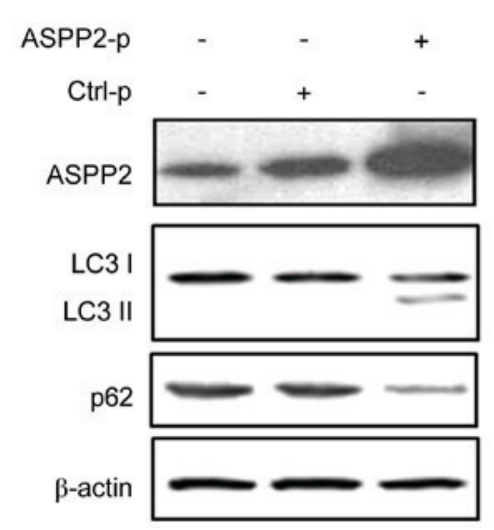

Figure 1. ASPP2 overexpression induces autophagy in Huh7.5 cells. (A and B) Huh7.5 cells were co-transfected with plasmids encoding ASPP2 (ASPP2-p) and GFP-LC3 for $48 \mathrm{~h}$. Control plasmid (Ctrl-p) is a control of ASPP2-p. (A) Immunofluorescence detection of GFP-LC3-II puncta in Huh7.5 cells. (B) The rate of puncta $^{+}$cells in (A) represents the mean \pm standard deviation of three independent experiments. (C) Hun7.5 cells were transfected with ASPP2-p or Ctrl-p for $48 \mathrm{~h}$. Western blot analysis detection of ASPP2, LC3-I/II, p62 with indicated antibodies. ASPP2, apoptosis-stimulating protein of p53; Ctrl-p, control plasmid; LC3-I/II, microtubule-associated proteins 1A/1B light chain 3A/3B; p62, sequestosome-1; GFP, green fluorescent protein.

Huh7.5 cells with or without transfection with ASPP2-p. It was determined that 3-MA treatment successfully inhibited basal and ASPP2-induced autophagy in Huh7.5 cells as demonstrated by a significant decrease of GFP-LC3-II puncta-positive cells (Fig. 4A). Next, TUNEL and Calcein AM/PI assays determined that ASPP2 overexpression significantly induced apoptosis and cell death when autophagy was inhibited by 3-MA treatment in Huh7.5 cells (Fig. 4B). These data indicated that, at least in Huh7.5 cells, autophagy impaired the function of ASPP2 overexpression on inducing apoptotic cell death.

Overexpression of DRAM recovers the function of ASPP2 on inducing apoptotic cell death in Huh7.5 cells. It was previously demonstrated that the expression of DRAM could give autophagy the ability to induce apoptosis (4). In the present study, Huh7.5 cells were co-transfected with plasmids encoding ASPP 2 and DRAM for $48 \mathrm{~h}$ to induce the overexpression of ASPP2 and DRAM (Fig. 5A). The results of the TUNEL and Calcein AM/PI assays revealed that overexpression of ASPP2 and DRAM significantly induced apoptotic cell death in Huh7.5 cells, indicating that overexpression of DRAM recovered the pro-apoptotic function of ASPP2 (Fig. 5B). These data indicated that induction of DRAM expression could improve the function of ASPP2 on inducing apoptosis, which may aid the treatment of HCC.

\section{Discussion}

ASPP2, an inducer of apoptosis, has been reported to inhibit the growth of hepatoma cells in vitro and in vivo (9). The present study demonstrated that ASPP2 overexpression failed to induce apoptosis in Huh7.5 cells, as ASPP2-induced autophagy impaired the function of ASPP2 on inducing apoptosis. These results are different from previous results, which demonstrated that ASPP2 overexpression-induced autophagy could induce apoptosis in HCC Hep1-6, HepG2 and Hep3B cell lines in a CHOP- and DRAM-dependent manner. We hypothesize that the different background of $\mathrm{HCC}$ cell lines is a critical factor that affects the function of ASPP2-induced autophagy on inducing or inhibiting apoptosis.

Previous studies demonstrated that ASPP2 is an autophagy inhibitor that impairs the formation of the autophagosome membrane by interacting with Atg5 $(10,11)$. However, a previous study also demonstrated that ASPP2 could induce autophagy development by increasing CHOP expression. $\mathrm{CHOP}$ reduces the level of $\mathrm{B}$-cell lymphoma-2 (Bcl-2) and then reduces the formation of Bcl-2-Beclin-1 complexes, which contributes to the increase of free Beclin-1 in the cytoplasm and the initiation of autophagy (4). In the present study, although CHOP is not involved in ASPP2-induced autophagy, 
A

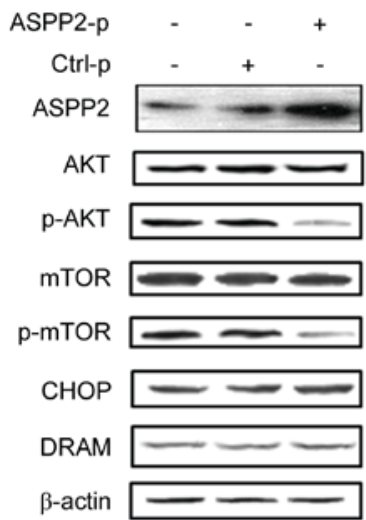

$\beta$-actin


C

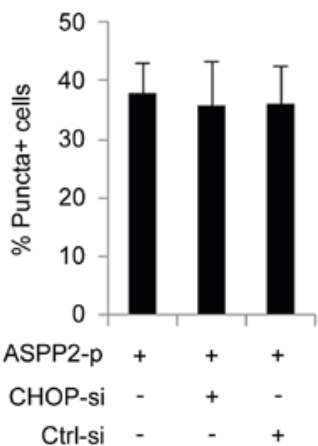

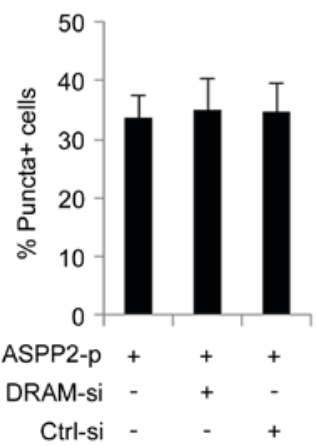

Figure 2. ASPP2 overexpression inactivates the AKT/mTOR pathway and CHOP/DRAM has no effect on ASPP2-induced autophagy. (A) Hun7.5 cells were transfected with ASPP2-p or Ctrl-p for $48 \mathrm{~h}$. Western blotting detection with indicated antibodies. (B) Huh7.5 cells were co-transfected with siRNAs to knock down expression of CHOP/DRAM (upper panel, CHOP-si; lower panel, DRAM-si) and ASPP2-p for $48 \mathrm{~h}$. Western blotting was used to detect the effect of siRNAs treatment on knocking down of CHOP and DRAM. (C) Huh7.5 cells were co-transfected with siRNAs to knock down CHOP/DRAM (left panel, CHOP-si; right panel, DRAM-si) and ASPP2-p for $48 \mathrm{~h}$. Immunofluorescence assay was used to detect the level of GFP-LC3-II-positive cells. The values represent the mean \pm standard deviation of three independent experiments. ASPP2, apoptosis-stimulating protein of p53; AKT, RAC serine/threonine-protein kinase; mTOR, mechanistic target of rapamycin; Ctrl-p, control plasmid; siRNA, small interfering RNA; CHOP, C/EBP homologous protein; DRAM, DNA damage regulated autophagy modulator 1; CHOP-si, siRNA targeting CHOP; GFP-LC3-II, green fluorescent protein-tagged microtubule-associated protein 1A/1B light chain 3B.

A



B

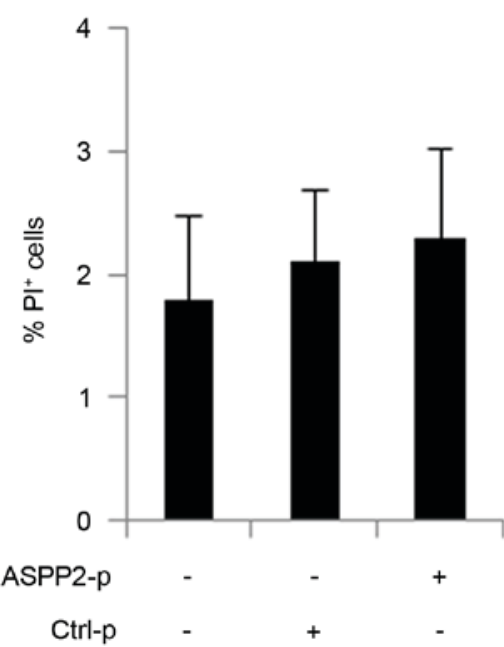

Figure 3. ASPP2 overexpression fails to induce apoptotic cell death in Huh7.5 cells. (A) TUNEL and (B) Calcein AM/PI were used to detect the levels of apoptosis and cell death in Huh7.5 cells transfected with ASPP2-p for $48 \mathrm{~h}$, respectively. The values represent the mean \pm standard deviation of three independent experiments. ASPP2, apoptosis-stimulating protein of p53; TUNEL, terminal deoxynucleotidyl transferase dUTP nick-end labeling; AM, acetoxymethyl ester; PI, propidium iodide 
A

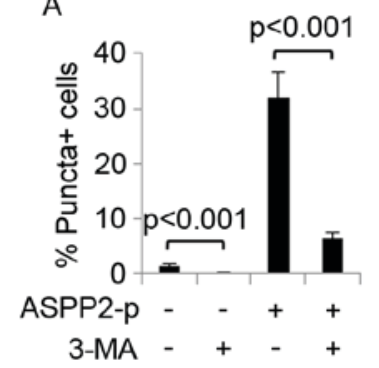

A

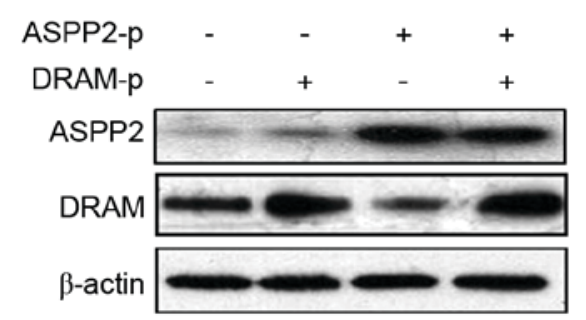

B

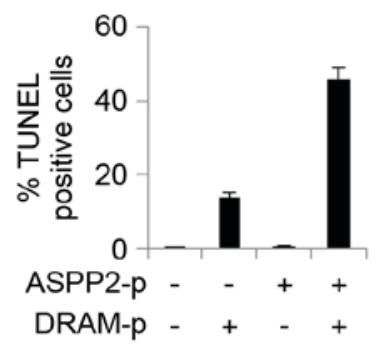

Figure 4. Autophagy impairs the function of ASPP2 on inducing apoptosis Huh7.5 cells were treated with autophagy inhibitor 3-MA and transfected with ASPP2-p. (A) Immunofluorescence assay was used to detect the effect of 3-MA on inhibiting ASPP2-induced autophagy. (B) TUNEL and Calcein AM/PI assays were used to detect the effect of autophagy inhibition via 3-MA on ASPP2-induced apoptosis and cell death. The values represent the mean \pm standard deviation of three independent experiments. ASPP2, apoptosis-stimulating protein of p53; TUNEL, terminal deoxynucleotidyl transferase dUTP nick-end labeling; AM, acetoxymethyl ester; PI, propidium iodide; 3-MA, 3-methyladenosine; ASPP2-p, ASPP2 plasmid.

it was identified that inactivation of the AKT/mTOR pathway also contributes to ASPP2-induced autophagy, indicating that in different situations ASPP2 can induce autophagy through activation of different mechanisms.

DRAM has been identified to induce apoptosis in an autophagy dependent or independent manner (4). In the present study, although DRAM is not involved in ASPP2-induced autophagy, overexpression of DRAM recovers the function of ASPP2 on inducing apoptotic cell death in Huh7.5 cells. To the best of our knowledge, the mechanism by which DRAM induces apoptosis or autophagic apoptosis remains unclear; however, the data in the present study strongly indicated that elucidation of the mechanisms by which DRAM induces apoptosis is critical for treating tumors $(4,12)$.

Autophagy is regarded to serve dual roles on cell death. Autophagy is reported to prevent cells from cell death signals, including nutrition depletion or organelle damage (5). Autophagy could degrade certain cytoplasmic redundant organelle or proteins to provide nutrition for cells (5). The autophagy-mediated degradation of impaired organelles, including uncoupled mitochondria, eliminates the large production of pro-apoptotic inducers, preventing pro-apoptotic factor-initiated cell death (13). However, other studies have demonstrated that autophagy can also be a pro-apoptotic factor, since the inhibition of autophagy reduces the level of apoptosis and the promotion of autophagy has an opposite result $(4,12,14)$. In the present study, ASPP2-induced autophagy had an anti-apoptotic role and the induction of autophagy was associated with the inactivation of the AKT/mTOR pathway.

Figure 5. Overexpression of DRAM recovered the pro-apoptotic function of ASPP2 in Huh7.5 cells. Huh7.5 cells were transfected with plasmids encoding ASPP2 or DRAM, or co-transfected with the two plasmids for $48 \mathrm{~h}$. (A) Western blotting detection of overexpression of ASPP2 and DRAM. (B) TUNEL (left panel) and Calcein AM/PI (right panel) assays were used to detect apoptosis and cell death, respectively. The values represent the mean \pm standard deviation of three independent experiments. ASPP2, apoptosis-stimulating protein of p53; TUNEL, terminal deoxynucleotidyl transferase dUTP nick-end labeling; AM, acetoxymethyl ester; PI, propidium iodide; DRAM, DNA damage regulated autophagy modulator 1.

In fact, although autophagy and apoptosis are involved in maintaining cellular homeostasis and the two physiological functions are regarded to be closely associated with each other, the mechanism by which autophagy induces apoptosis remains, to the best of our knowledge, unclear. The data generated in the present study indicated that the different autophagy-inducing signals may determine the different roles of autophagy on apoptosis; for example, CHOP/DRAM-induced autophagy induces apoptosis, but inactivation of AKT/mTOR pathway induces an anti-apoptotic autophagy.

mTOR is a major target of AKT. When mTOR is activated by AKT via phosphorylation, activated mTOR will inhibit autophagy (15). Previous studies indicate that inhibition of mTOR function by its inhibitors promotes autophagy development and reduces the sensitivity of cells to cell death signals $(16,17)$. Up to now, the mechanism by which ASPP2 inactivates the AKT/mTOR pathway remains unclear, because, to the best of our knowledge, few studies have investigated the associated between the AKT/mTOR pathway and ASPP2. A previous study demonstrated that ASPP2 could bind to AKT in nucleus (3). In the present study, although it is not known whether the interaction between of ASPP2 and AKT could affect the activation of AKT, the data indicated that ASPP2 had the ability to suppress the function of AKT, and overexpression of ASPP2 might be benefit for treating certain tumors with high AKT activation levels.

Taken together, the results of the present study revealed a mechanism by which hepatoma cells could escape from ASPP2-induced apoptosis. These data may prove valuable for the future study of tumor therapy. 


\section{Acknowledgements}

Not applicable.

\section{Funding}

The present study was supported by grants from National Natural Science Foundation of China (grant nos. 81402556, 81773168 and 81272266), International Cooperation and Exchanges NSFC (no. 81361120401), The capital health research and development of special foundation (no. 2014-1-1151), and the foundation of Beijing Institute of Hepatology (nos. BJIH-01602 and BJIH-01715), Tumor Invasion and Metastasis Mechanism Research Key Laboratory Open Fund of Beijing2015 (no. 2015ZLQX05).

\section{Availability of data and materials}

All data generated or analyzed during this study are included in this published article.

\section{Authors' contributions}

DL and RL contributed equally to this work, both completed the majority of the laboratory work. XG and LP assisted DL and RL to complete the rest of the laboratory work. DC and KL designed the project. YZ assisted DC and KL to design the experimental work. KL completed the writing of this article.

\section{Ethics approval and consent to participate}

Not applicable.

\section{Consent for publication}

Not applicable.

\section{Competing interests}

The authors declare that they have no competing interests.

\section{References}

1. Bergamaschi D, Samuels Y, O'Neil NJ, Trigiante G, Crook T, Hsieh JK, O'Connor DJ, Zhong S, Campargue I, Tomlinson ML, et al: iASPP oncoprotein is a key inhibitor of p53 conserved from worm to human. Nat Genet 33: 162-167, 2003.
2. Song B, Bian Q, Zhang YJ, Shao CH, Li G, Liu AA, Jing W, Liu R, Zhou YQ, Jin G and Hu XG: Downregulation of ASPP2 in pancreatic cancer cells contributes to increased resistance to gemcitabine through autophagy activation. Mol Cancer 14: 177, 2015.

3. Liu K, Jiang T, Ouyang Y, Shi Y, Zang Y, Li N, Lu S and Chen D: Nuclear EGFR impairs ASPP2-p53 complex-induced apoptosis by inducing SOS1 expression in hepatocellular carcinoma. Oncotarget 6: 16507-16516, 2015.

4. Liu K, Shi Y, Guo X, Wang S, Ouyang Y, Hao M, Liu D, Qiao L, Li N, Zheng J and Chen D: CHOP mediates ASPP2-induced autophagic apoptosis in hepatoma cells by releasing Beclin-1 from Bcl-2 and inducing nuclear translocation of Bcl-2. Cell Death Dis 5: e1323, 2014.

5. Shirakabe A,Ikeda Y, Sciarretta S, Zablocki DK and Sadoshima J: Aging and autophagy in the heart. Circ Res 118: 1563-1576, 2016.

6. Lin L and Baehrecke EH: Autophagy, cell death, and cancer. Mol Cell Oncol 2: e985913, 2015.

7. Liu K, Lou J, Wen T, Yin J, Xu B, Ding W, Wang A, Liu D, Zhang C, Chen D and Li N: Depending on the stage of hepatosteatosis, p53 causes apoptosis primarily through either DRAM-induced autophagy or BAX. Liver Int 33: 1566-1574, 2013.

8. Tan VP and. Miyamoto S: Nutrient-sensing mTORC1: Integration of metabolic and autophagic signals. J Mol Cell Cardiol 95: 31-41, 2016.

9. Zhao J, Wu G, Bu F, Lu B, Liang A, Cao L, Tong X, Lu X, Wu M and Guo Y: Epigenetic silence of ankyrin-repeat-containing, SH3-domain-containing, and proline-rich-region-containing protein 1 (ASPP1) and ASPP2 genes promotes tumor growth in hepatitis B virus-positive hepatocellular carcinoma. Hepatology 51: 142-153, 2010.

10. Wang Y, Wang XD,Lapi E, Sullivan A, Jia W,He YW, Ratnayaka I, Zhong S, Goldin RD, Goemans CG, et al: Autophagic activity dictates the cellular response to oncogenic RAS. Proc Natl Acad Sci USA 109: 13325-13330, 2012.

11. Chen R, Wang H, Liang B, Liu G, Tang M, Jia R, Fan X, Jing $\mathrm{W}$, Zhou X, Wang H, et al: Downregulation of ASPP2 improves hepatocellular carcinoma cells survival via promoting BECN1-dependent autophagy initiation. Cell Death Dis 7: e2512, 2016.

12. Liu K, Shi Y, Guo XH, Ouyang YB, Wang SS, Liu DJ, Wang AN, Li N and Chen DX: Phosphorylated AKT inhibits the apoptosis induced by DRAM-mediated mitophagy in hepatocellular carcinoma by preventing the translocation of DRAM to mitochondria. Cell Death Dis 5: e1078, 2014.

13. Flores-Toro JA, Go KL, Leeuwenburgh C and Kim JS: Autophagy in the liver: Cell's cannibalism and beyond. Arch Pharm Res 39: 1050-1061, 2016.

14. Rodríguez ME, Cogno IS, Milla Sanabria LS, Morán YS and Rivarola VA: Heat shock proteins in the context of photodynamic therapy: Autophagy, apoptosis and immunogenic cell death. Photochem Photobiol Sci 15: 1090-1102, 2016.

15. Chagin AS: Effectors of mTOR-autophagy pathway: Targeting cancer, affecting the skeleton. Curr Opin Pharmacol 28: 1-7, 2016.

16. Xu L and Brink M: mTOR, cardiomyocytes and inflammation in cardiac hypertrophy. Biochim Biophys Acta 1863: 1894-1903, 2016.

17. Zheng XT, Wu ZH, Wei Y, Dai JJ, Yu GF, Yuan F and Ye LC: Induction of autophagy by salidroside through the AMPK-mTOR pathway protects vascular endothelial cells from oxidative stress-induced apoptosis. Mol Cell Biochem 425: 125-138, 2017. 